\title{
Effect of Prepregnancy Lymphocyte Active Immunotherapy on Unexplained Recurrent Miscarriage, Pregnancy Success Rate, and Maternal-Infant Outcome
}

\author{
Junxia Li, Yan Gu, Shaojing Zhang, Baohui Ju, and Jianmei Wang \\ Department of Obstetrics and Gynecology, Second Hospital Affiliated to Tianjin Medical University, China \\ Correspondence should be addressed to Jianmei Wang; wangjianmei666666@126.com
}

Received 24 June 2021; Accepted 20 September 2021; Published 14 October 2021

Academic Editor: A.Seval Ozgu-Erdinc

Copyright (c) 2021 Junxia Li et al. This is an open access article distributed under the Creative Commons Attribution License, which permits unrestricted use, distribution, and reproduction in any medium, provided the original work is properly cited.

\begin{abstract}
Objective. To evaluate the effect of prepregnancy lymphocyte active immunotherapy on unexplained recurrent miscarriage, pregnancy success rate, and maternal-infant outcome. Methods. A total of 124 patients with recurrent miscarriage admitted to our hospital from January 2018 to December 2020 were selected as the research objects and divided into the experimental group and the control group according to the random number table method, with 62 patients in each group. The experimental group was treated with lymphocyte active immunotherapy, and the control group was given conventional treatment. The pregnancy success rate, estrogen indexes, hemorheology indexes, and psychological state of the two groups were compared. Results. The experimental group garnered a notably higher pregnancy success rate and a prominently lower miscarriage rate than the control group $(P<0.05)$. Better results of self-rating anxiety scale (SAS) and self-rating depression scale (SDS) were observed in the experimental group, as compared to the control group $(P<0.05)$. The experimental group yielded more desirable results in terms of treatment satisfaction, estrogen indexes, and hemorheology indexes in comparison with the control group $(P<0.05)$. Conclusion. The use of lymphocyte active immunotherapy for patients with unexplained recurrent miscarriage can significantly increase the pregnancy success rate, optimize the maternal-infant outcome, drive down the miscarriage rate, and ameliorate the patient's estrogen levels and hemorheology indicators, which is worthy of promotion and application in clinical practice.
\end{abstract}

\section{Introduction}

The spontaneous miscarriage that occurs twice or more in a row is called recurrent miscarriage, and that occurs three times or more in a row is habitual miscarriage. Only 50\% of patients with recurrent miscarriage can be diagnosed with specific causes which mainly include unhealthy lifestyle, maternal endocrine abnormalities, maternal reproductive tract abnormalities, cervical insufficiency, chromosomal abnormalities, reproductive tract infections, and chromosomal abnormalities, while the cause of the other $50 \%$ of patients still remains not clear [1-3]. Relevant studies in recent years have shown that more than half of the causes of unexplained recurrent miscarriage, which has been witnessed an increasing trend recently, are closely related to immune dysfunction, that is, imbalance of pregnancy immune tolerance and abnormal maternal immune regulation. To date, consensus on the treatment of unexplained recurrent miscarriage has not yet been developed. Despite the safety and effectiveness of lymphocyte active immunotherapy are challenged at times, it is the most common therapy in clinical practice [4-6]. Therefore, this study is to assess the effect of prepregnancy lymphocyte active immunotherapy on unexplained recurrent miscarriage, pregnancy success rate, and maternal-infant outcome. The report is as follows:

\section{Materials and Methods}

2.1. General Information. A total of 124 patients with recurrent miscarriage admitted to our hospital from January 2018 
TABLE 1: Comparison of clinical information between the two groups.

\begin{tabular}{|c|c|c|c|c|}
\hline Categories & Experimental group $(n=62)$ & Control group $(n=62)$ & $\chi^{2} / t$ & $P$ \\
\hline Mean age (year) & $27.34 \pm 2.31$ & $27.37 \pm 2.34$ & 0.072 & 0.943 \\
\hline Mean height $(\mathrm{cm})$ & $162.45 \pm 3.42$ & $162.51 \pm 3.44$ & 0.097 & 0.923 \\
\hline Mean pregnancy times & $2.32 \pm 0.34$ & $2.34 \pm 0.36$ & 0.318 & 0.751 \\
\hline \multicolumn{5}{|l|}{ Education level } \\
\hline University & $31(50.00 \%)$ & $29(46.77 \%)$ & 0.129 & 0.719 \\
\hline Middle school & $23(37.10 \%)$ & $24(38.71 \%)$ & 0.034 & 0.853 \\
\hline Primary school & $8(12.90 \%)$ & $9(14.52 \%)$ & 0.068 & 0.794 \\
\hline Place of residence & & & 0.134 & 0.714 \\
\hline Urban & $24(38.71 \%)$ & $26(41.94 \%)$ & & \\
\hline Rural & $38(61.29 \%)$ & $36(58.06 \%)$ & & \\
\hline
\end{tabular}

to December 2020 were selected as the research objects and divided into the experimental group and the control group according to the random number table method, with 62 patients in each group.

2.2. Inclusion Criteria. (1) All patients met with the diagnostic criteria for unexplained recurrent miscarriage; (2) the chromosomal examinations of all couples were normal; (3) the endocrine function of the patients was normal; (4) the test results of anti-cardiolipin, anti-endometrial, anti-nuclear, and other antiautoantibodies of all patients were negative. (5) This study was approved by the hospital ethics committee. The patients and their families signed an informed consent form after being fully informed of the purpose and process of the study.

2.3. Exclusion Criteria. (1) The liver function test results of the couples were abnormal; (2) the patients had reproductive tract infections such as chlamydia, HIV, mycoplasma, and cytomegalovirus; (3) the semen examination of the patients' husband or a third party who provided lymphocytes was abnormal; (4) B-ultrasound examination showed that the patients' uterus had congenital malformations and intrauterine adhesions.

2.4. Methods. The control group received conventional treatment, given progesterone (National Medical Product Administration Approval Number H20041902; Zhejiang Xianju Pharmaceutical Co., Ltd.; $50 \mathrm{mg} * 10 \mathrm{~s} * 2$ plate), once/day, $100 \mathrm{mg} /$ time, after meals, for 14 days. When the patient was diagnosed with pregnancy, intramuscular injection of human chorionic gonadotropin $2000 \mathrm{U}$ was performed, $2 \mathrm{~d}$ /time. After a week of continuous injection, it was then changed to 2 times/week, and after two weeks of continuous injection, it was changed to 1 time/week. The treatment continued for 3 months.

The experimental group received lymphocyte active immunotherapy: (1) before active immunization, a blocking antibody test should be performed on empty stomach during pregnancy. $100 \mu \mathrm{l}$ of the patient's husband's peripheral anticoagulated whole blood was collected, $20 \mu$ l of mouse anti-human CD3, CD4, and CD8 monoclonal antibodies was added, and then, $50 \mu \mathrm{l}$ of patient serum or normal male
TABLE 2: Comparison of pregnancy success rate between the two groups after treatment $(n(\%))$.

\begin{tabular}{lccc}
\hline Groups & $n$ & $\begin{array}{c}\text { Pregnancy success } \\
\text { rate }\end{array}$ & $\begin{array}{c}\text { Miscarriage } \\
\text { rate }\end{array}$ \\
\hline Experimental group & 62 & $59(95.16)$ & $3(4.84)$ \\
Control group & 62 & $47(75.81)$ & $15(24.19)$ \\
$\chi^{2}$ & & & 9.359 \\
$P$ & & & 0.002 \\
\hline
\end{tabular}

$\mathrm{AB}$ serum was added, incubated for 30 minutes; next, $3 \mathrm{ml}$ of red blood cell lysis solution was added and washed twice with PBS; subsequently, a flow cytometry was used to analyze the ratio of CD3, CD4, and CD8 cells of spouse or third male lymphocytes with female serum and $\mathrm{AB}$ serum, and the blocking antibody efficiency was calculated according to the following formula: blocking efficiency $=\mathrm{CD}$ ratio with $\mathrm{AB}$ serum $(\%)$ - the proportion of female serum $\mathrm{CD}(\%)$, the normal fertility group blocking efficiency is X-1.96s, and those greater than this value is considered positive. If the test result was negative, the peripheral blood would be drawn again after 4 times of immunotherapy, and the blocking antibodies in the body would be checked in the same way [7]. (2) During the active immunization process, the couples were genotyped with sequence-specific primer PCR for HLAII locus. The husband's lymphocytes are the first choice for active immunization. If the husband is not suitable as an immunogen donor (HBsAg positive), other healthy individuals should be selected. The consistency of the two sites indicated an involvement of a third party donor, and a blood type test for the both parties was performed. Subsequently, $20 \mathrm{ml}$ of the donor's peripheral blood was drawn, and the lymphocytes were separated by Ficoll density gradient centrifugation and washed with sterile saline three times to remove platelets and form a lymphocyte suspension with a concentration of $(2 \sim 4) \times 107 / \mathrm{mL}$. After disinfection of the skin on the inner forearm, 2 to 4 points were selected for subcutaneous injection $(0.5 \mathrm{~cm}$ for the interval), with $0.2 \sim 0.3 \mathrm{~mL}$ each time at each point, 3 weeks/time, and a course of treatment contained 4 times of treatment. Contraception was indispensable during the treatment. After one course of 


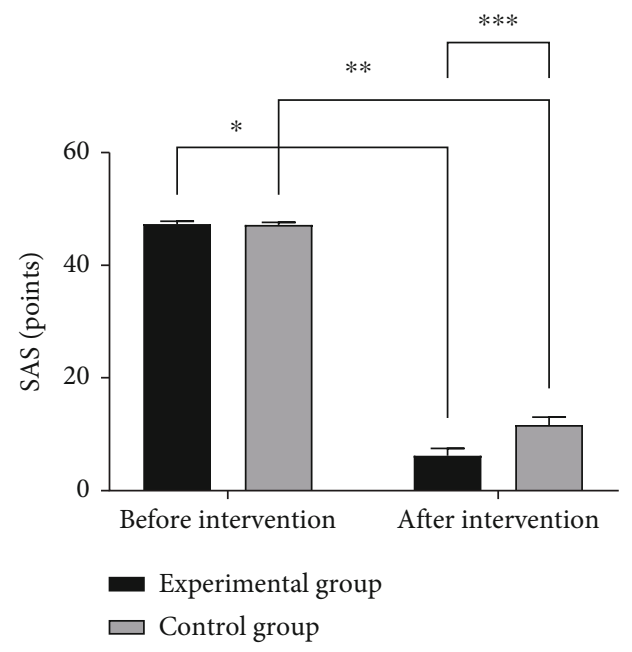

Figure 1: Comparison of SAS scores between the two groups before and after treatment $(\bar{x} \pm s)$. Note: the abscissa represents the experimental group and control group after treatment, and the ordinate represents the SAS scores, points. The SAS scores of the experimental group before and after treatment are $47.33 \pm 0.51$ points and $6.21 \pm 1.33$ points, respectively. The SAS scores of the control group before and after treatment are $47.17 \pm 0.48$ points and $11.66 \pm 1.43$ points, respectively; $*$ indicates that the SAS scores of patients in the experimental group before and after treatment are significantly different $(t=227.304, P \leq 0.01) ; * *$ indicates that the SAS scores of patients in the experimental group before and after treatment are significantly different $(t=185.365, P \leq 0.01) ; * * *$ indicates that the SAS scores of the two groups of patients after treatment are significantly different $(t=21.974, P \leq 0.01)$.

treatment, if the test result of blocking antibodies was positive, the patient would be advised to conceive within three months; otherwise, lymphocyte immunotherapy would be continued till the test result turned into positive, and the patient then was permitted to get pregnant. The therapy lasted for 3 months $[8,9]$.

After the treatment, a six-month follow-up was carried out. The adverse reactions of the patients were recorded in detail and handled with timely and effective treatment.

\subsection{Indicators Observation. The pregnancy success rate of} the two groups was compared.

The "Patient Clinical Satisfaction Survey Questionnaire" prepared by the hospital was used to investigate the patients' satisfaction with treatment. The degree of satisfaction was divided into satisfied, basically satisfied, and dissatisfied. Total satisfaction $=$ satisfied + basically satisfied.

Radioimmunoassay was used to measure the changes of estrogen indicators in the two groups of patients. The estrogen indicators contain progesterone, estradiol, and $\beta$-HCG.

Flow cytometer, viscometer, and electrophoresis instrument were used to detect hemorheology indexes of the two groups of patients. Hemorheology indexes include hematocrit (HCT), plasma viscosity (PV), erythrocyte sedimentation rate (ESR), and erythrocyte electrophoresis time (EET).

The SAS [10] was used to assess the psychological anxiety of the two groups of patients before and after interven-

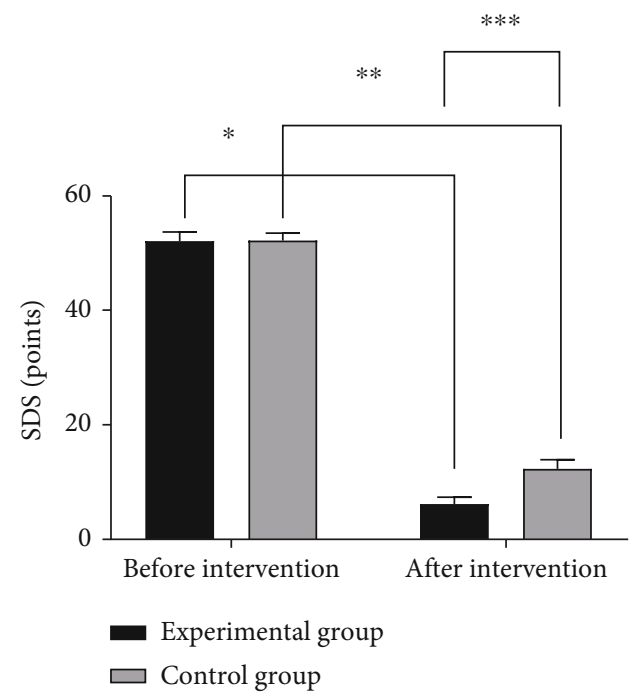

FIgURE 2: Comparison of SDS scores between the two groups $(\bar{x} \pm s)$. Note: the abscissa represents the experimental group and control group after treatment, and the ordinate represents the SDS scores, points. The SDS scores of the experimental group before and after treatment are $52.13 \pm 1.6$ points and $6.18 \pm 1.2$ points, respectively. The SDS scores of the control group before and after treatment are $52.21 \pm 1.3$ points and $12.33 \pm 1.6$ points, respectively; $*$ indicates that the SDS scores of patients in the experimental group before and after treatment are significantly different $(t=180.905, P \leq 0.01) ; * *$ indicates that the SDS scores of patients in the experimental group before and after treatment are significantly different $(t=152.320, P \leq 0.01) ; * * *$ indicates that the SDS scores of patients in the experimental group before and after treatment are significantly different $(t=24.213, P \leq 0.01)$.

tion. The total score of the scale is 50 points. The higher the score, the more serious the patient's anxiety.

The SDS [11] was used to evaluate the depression of the two groups of patients before and after the intervention. The total score of the scale is 55 points. The higher the score, the more severe the depression of the patient.

2.6. Statistical Processing. The experimental data was statistically analyzed and processed by the SPSS21.0 software, and GraphPad Prism 7 (GraphPad Software, San Diego, USA) was used to plot graphics. The count data was represented by $n$ (\%), using $\chi^{2}$ test, and the measurement data was expressed as $\bar{x} \pm s$, using $t$-test. A $P$ value less than 0.05 was considered statistically significant.

\section{Results}

3.1. Comparison of Clinical Information between the Two Groups. The two groups did not show great disparity in terms of their general information such as mean age, mean height, mean pregnancy times, education level, and place of residence $(P>0.05)$ (see Table 1$)$.

3.2. Comparison of Pregnancy Success Rate between the Two Groups after Treatment. Table 2 demonstrates a higher pregnancy success rate and a lower miscarriage rate of the experimental group than that of the control group $(P<0.05)$. 


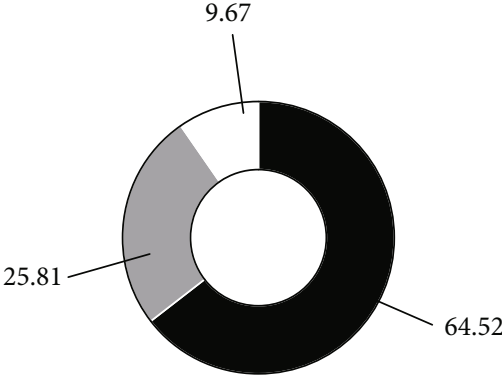

Total $=100$

Experimental group

- Satisfaction

$\square$ More satisfied

$\square$ Dissatisfied

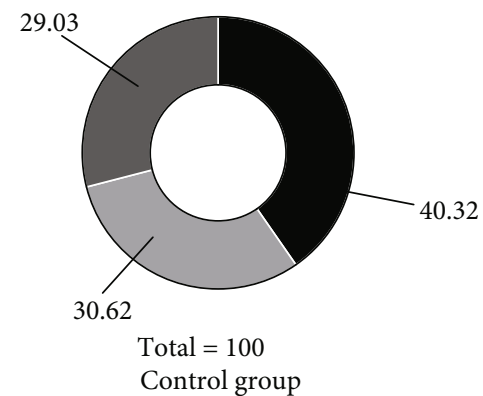

Satisfaction

More satisfied

$\square$ Dissatisfied

(a)

(b)

Figure 3: Comparison of satisfaction between the two groups ( $n(\%)$ ). Note: (a) the treatment effect in the experimental group; (b) the treatment effect in the control group. The satisfied rate of the experimental group is $64.52 \%(40 / 62)$, the basically satisfied rate is $25.81 \%$ $(16 / 62)$, the dissatisfied rate is $9.67 \%(6 / 62)$, and the overall satisfaction rate is $90.32 \%(56 / 62)$. The satisfied rate of the control group is $40.32 \%(25 / 62)$, the basically satisfied rate is $30.65 \%(19 / 62)$, the dissatisfied rate is $29.03 \%(18 / 62)$, and the overall satisfaction rate is $75.81 \%(47 / 62)$. There is significant difference between the two groups of patients after treatment $\left(\chi^{2}=6.719, P=0.010\right)$.

TABLE 3: Comparison of estrogen indicators between the two groups of patients $(\bar{x} \pm s)$.

\begin{tabular}{lccccccc}
\hline \multirow{2}{*}{ Groups } & \multirow{2}{*}{$n$} & \multicolumn{2}{c}{ Estradiol } & \multicolumn{2}{c}{$\beta$-HCG } & \multicolumn{2}{c}{ Progesterone } \\
& & Before treatment & After treatment & Before treatment & After treatment & Before treatment & After treatment \\
\hline Experimental group & 62 & $62.45 \pm 8.37$ & $139.74 \pm 20.48$ & $14.26 \pm 2.04$ & $24.09 \pm 4.37$ & $22.13 \pm 2.81$ & $32.31 \pm 5.53$ \\
Control group & 62 & $62.54 \pm 8.43$ & $113.27 \pm 46.74$ & $14.37 \pm 2.01$ & $18.73 \pm 3.21$ & $22.26 \pm 2.86$ & $27.43 \pm 4.41$ \\
$t$ & & 0.060 & 4.084 & 0.302 & 7.784 & 0.255 & 5.433 \\
$P$ & & 0.953 & $\leq 0.01$ & 0.763 & $\leq 0.01$ & 0.799 & $\leq 0.01$ \\
\hline
\end{tabular}

TABLE 4: Comparison of hemorheology indexes between the two groups of patients $(\bar{x} \pm s)$.

\begin{tabular}{lccccccccc}
\hline Groups & $n$ & \multicolumn{2}{c}{ HCT } & \multicolumn{2}{c}{ EEP } & & ERP & & PV \\
& & $\begin{array}{c}\text { Before } \\
\text { treatment }\end{array}$ & $\begin{array}{c}\text { After } \\
\text { treatment }\end{array}$ & $\begin{array}{c}\text { Before } \\
\text { treatment }\end{array}$ & $\begin{array}{c}\text { After } \\
\text { treatment }\end{array}$ & $\begin{array}{c}\text { Before } \\
\text { treatment }\end{array}$ & $\begin{array}{c}\text { After } \\
\text { treatment }\end{array}$ & $\begin{array}{c}\text { Before } \\
\text { treatment }\end{array}$ & $\begin{array}{c}\text { After } \\
\text { treatment }\end{array}$ \\
\hline Experimental group & 62 & $38.17 \pm 5.13$ & $25.66 \pm 3.74$ & $348.51 \pm 22.11$ & $293.12 \pm 12.54$ & $28.18 \pm 6.23$ & $15.19 \pm 4.57$ & $2.44 \pm 0.57$ & $1.23 \pm 0.34$ \\
Control group & 62 & $38.21 \pm 5.07$ & $32.43 \pm 4.54$ & $349.46 \pm 21.87$ & $325.18 \pm 18.68$ & $28.27 \pm 6.34$ & $21.73 \pm 5.84$ & $2.47 \pm 0.61$ & $1.72 \pm 0.41$ \\
$t$ & & 0.044 & 9.063 & 0.241 & 11.220 & 0.080 & 6.944 & 0.283 & 7.244 \\
$P$ & 0.965 & $\leq 0.01$ & 0.810 & $\leq 0.01$ & 0.937 & $\leq 0.01$ & 0.778 & $\leq 0.01$ \\
\hline
\end{tabular}

3.3. Comparison of SAS Scores between the Two Groups before and after Treatment. The experimental group yielded a superior outcome of SAS scores when compared with the control group $(P<0.05)$ (see Figure 1$)$.

3.4. Comparison of SDS Scores between the Two Groups before and after Treatment. The SDS scores of the experimental group after intervention were significantly better than that of the control group $(P<0.05)$, as shown in Figure 2 .

3.5. Comparison of Treatment Satisfaction between the Two Groups. Patients in the experimental group were more satisfied with the treatment than those in the control group $(P<0.05)$, as shown in Figure 3.

3.6. Comparison of Estrogen Indicators between the Two Groups of Patients. Strong evidence of higher estrogen indicators of the experimental group by contrast to the control group was found $(P<0.05)$ (see Table 3$)$.

3.7. Comparison of Hemorheology Indexes between the Two Groups of Patients. Table 4 presents a significant difference in hemorheology indicators between the experimental group and the control group after treatment $(P<0.05)$. 


\section{Discussion}

At present, the current childbearing age is generally delayed with the change of people's life concept, and a gradual rise in the incidence of recurrent miscarriage has been witnessed year by year. Recurrent miscarriage is considered as a refractory disease in consequence of its rather complicated causes in clinic, among which the miscarriage triggered by immune factors accounts for more than half [12-15]. In modern immunology, it is believed that the genetic antigens from the father during the embryo formation process are treated as foreign invaders, which triggers rejection reactions in the mother; hence, miscarriage indicates the failure of semihomogenous transplantation. The body of normal pregnant women will produce blocking antibodies on their own to fight against natural rejection, thus ensuring the growth and development of the embryo. However, the deficiency of blocking antibodies in the mother will consequently lead to the attack from the mother's natural rejection on the embryo which eventually results in an abnormal gestation [16-20]. Such shortage of blocking antibodies can be remedied by the implantation of lymphocyte active immunotherapy into the patient's body through allogeneic lymphocytes to stimulate the patient's immune system. When the patient is pregnant again, the blocking antibodies that have been produced can be recognized by the homologous antibodies to ensure the protection of the embryo growth. Early recurrent miscarriage may also be related to the patient's low response to embryos and semihomogenous antigens, embryo rejection, and inability to produce suitable blocking antibodies. The deficiency of blocking antibodies is one of the main reasons behind recurrent miscarriage and the termination of embryo development. Studies have shown that more than $85 \%$ of terminated development of embryos and recurrent miscarriage are linked to the shortage of blocking antibodies in patients [21-24]. The use of lymphocyte immunotherapy can substantially elevate the transformation rate of blocking antibodies in patients from negative to positive. The results of this study showed prominently higher levels of estrogen indicators of the experimental group than those of the control group $(P<0.05)$, which was consistent with the research results of Salimi et al. [25] who pointed out that "after treatment, the indexes of estradiol, progesterone, and $\beta$-HCG in the experimental group were $138.81 \pm$ $20.43,32.33 \pm 5.51$, and $24.12 \pm 4.35$, respectively, and in the control group were $113.31 \pm 46.72,32.29 \pm 5.54$, and $24.09 \pm 4.37$, respectively. The estrogen indicators of the experimental group were significantly higher than those of the control group $(P<0.05)$." It is fully proved that the application of lymphocyte active immunotherapy for patients with unexplained recurrent miscarriage can predominantly optimize the estrogen indicators of patients.

In conclusion, prepregnancy lymphocyte active immunotherapy yields a promising therapeutic effect in the treatment of unexplained recurrent miscarriage. It not only ameliorates the patient's cellular immune function, but also further increases the patient's pregnancy success rate with a high safety, which is worthy of clinical application and promotion.

\section{Data Availability}

The data used to support the findings of this study are available from the corresponding author upon request.

\section{Conflicts of Interest}

The authors declare that they have no conflicts of interest.

\section{References}

[1] Y. Hou, J. Li, Q. Liu et al., "The optimal timing of immunotherapy may improve pregnancy outcome in women with unexplained recurrent pregnancy loss: a perspective followup study in northeastern China," American Journal of Reproductive Immunology, vol. 83, no. 4, 2020.

[2] H. Ou and Q. Yu, "Efficacy of aspirin, prednisone, and multivitamin triple therapy in treating unexplained recurrent spontaneous abortion: a cohort study," International Journal of Gynecology and Obstetrics: the official organ of the International Federation of Gynaecology and Obstetrics, vol. 148, no. 1, pp. 21-26, 2020.

[3] B. Liu, H. Wu, Q. Huang, M. Li, and X. Fu, "Phosphorylated STAT3 inhibited the proliferation and suppression of decidual Treg cells in unexplained recurrent spontaneous abortion," International Immunopharmacology, vol. 82, p. 106337, 2020.

[4] T. Wang, X. Kang, A. Zhao, L. He, Z. Liu, and F. Liu, "Lowdose aspirin improves endometrial receptivity in the midluteal phase in unexplained recurrent pregnancy loss," International Journal of Gynecology and Obstetrics: the official organ of the International Federation of Gynaecology and Obstetrics, vol. 150, no. 1, pp. 77-82, 2020.

[5] S. Hosseini, F. Shokri, S. A. Pour, J. Khoshnoodi, M. JeddiTehrani, and A.-H. Zarnani, "Diminished frequency of menstrual and peripheral blood NKT-like cells in patients with unexplained recurrent spontaneous abortion and infertile women," Reproductive Sciences, vol. 26, no. 1, pp. 97-108, 2019.

[6] D. D. Dean, S. Agarwal, and S. Muthuswamy, "Defining the role of FMR1 gene in unexplained recurrent spontaneous abortion," Journal of assisted reproduction and genetics, vol. 36, no. 11, pp. 2245-2250, 2019.

[7] Y. Gao, P. Wang, T. Zou, Z. Zhang, and W. Liang, "Increased Th17 and reduced Treg cells in patients with unexplained recurrent spontaneous abortion," Clinical and Experimental Obstetrics and Gynecology, vol. 46, no. 3, pp. 458-460, 2019.

[8] L. Yang, C. He, A. Gao, L. Xu, Z. Xu, and H. Zhang, "Elevated natural killer cells in the decidua and peripheral blood of patients with unexplained recurrent miscarriage," Journal of Reproductive Medicine, vol. 64, no. 1-2, pp. 38-44, 2019.

[9] L. Li, Q. Zhou, Y. R. Zhao, Y. L. Jiao, L. C. Wang, and S. Wang, "The distinctive variation in killer cell immunoglobulin-like receptor genotypes in patients with unexplained recurrent spontaneous abortions," Journal of Reproductive Medicine, vol. 64, no. 9-10, pp. 353-358, 2019.

[10] T. Nonaka, M. Tominaga, M. Takahashi, C. Nonaka, T. Enomoto, and K. Takakuwa, "Immunotherapy with paternal lymphocytes for patients with unexplained recurrent abortion and association with blocking antibodies comparison between patient groups with primary and secondary unexplained recurrent abortion," Journal of Reproductive Medicine, vol. 63, no. 5-6, pp. 285-291, 2018. 
[11] H. Motedayyen, A. H. Zarnani, N. Tajik, S. Ghotloo, and A. Rezaei, "Immunomodulatory effects of human amniotic epithelial cells on naive CD4 + T cells from women with unexplained recurrent spontaneous abortion," Placenta, vol. 71, pp. 7131-7140, 2018.

[12] G.-L. Xu, X. F. Hu, Y.-M. Han, and A.-W. Wei, "Clinical efficacy of low molecular heparin on unexplained recurrent spontaneous abortion," Clinical Laboratory, vol. 64, pp. 1037-1040, 2018.

[13] Y. Yang, L. Cheng, X. Deng, H. Yu, and L. Chao, "Expression of GRIM-19 in unexplained recurrent spontaneous abortion and possible pathogenesis," Molecular Human Reproduction, vol. 24, no. 7, pp. 366-374, 2018.

[14] D. Li, J. Li, B. Jia, Y. Wang, J. Zhang, and G. Liu, "Genomewide identification of microRNAs in decidual natural killer cells from patients with unexplained recurrent spontaneous abortion," AJRI: American Journal of Reproductive Immunology, vol. 80, no. 5, 2018.

[15] K. P. Muyayalo, Z. H. Li, G. Mor, and A. H. Liao, "Modulatory effect of intravenous immunoglobulin on Th17/Treg cell balance in women with unexplained recurrent spontaneous abortion," AJRI: American Journal of Reproductive Immunology, vol. 80, no. 4, article e13018, 2018.

[16] X. Zhao, Y. Jiang, L. Wang, Z. Li, Q. Li, and X. Feng, “Advances in understanding the immune imbalance between $\mathrm{T}$ lymphocyte subsets and NK cells in recurrent spontaneous abortion," Geburtshilfe und Frauenheilkunde, vol. 78, no. 7, pp. 677-683, 2018.

[17] L. Zhao, J. Li, and S. Huang, "Patients with unexplained recurrent spontaneous abortion show decreased levels of microrna146a-5p in the deciduae," Annals of Clinical and Laboratory Science: Official Journal of the Association of Clinical Scientists, vol. 48, no. 2, pp. 177-182, 2018.

[18] "Success rate of methotrexate treatment for recurrentvs.primary ectopic pregnancy: a case-control study," Journal of Obstetrics and Gynaecology: the journal of the Institute of Obstetrics and Gynaecology, vol. 40, no. 4, pp. 507-511, 2020.

[19] E. G. Aydeniz, U. Sari, and T. U. K. Dilek, "Pregnancy success rate at recurrent implantation failure patients after hysteroscopic endometrial injury: preliminary study," Clinical and Experimental Obstetrics and Gynecology, vol. 46, no. 5, pp. 709-712, 2019.

[20] A. A. Aboelroose, Z. M. Ibrahim, E. H. Madny, A. M. Elmazzahy, and O. T. Taha, "A randomized clinical trial of sildenafil plus clomiphene citrate to improve the success rate of ovulation induction in patients with unexplained infertility," International Journal of Gynecology and Obstetrics: the official organ of the International Federation of Gynaecology and Obstetrics, vol. 150, no. 1, pp. 72-76, 2020.

[21] M. Alalfy, A. Elgazzar, N. Ghamry et al., "Physical endometrial manipulation and its impact on success rate and live birth rate in ICSI in patients with unexplained infertility after recurrent ICSI failure, a double blinded randomized controlled trial," The journal of Maternal-Fetal \& Neonatal Medicine, vol. 33, no. 17, pp. 2983-2989, 2020.

[22] N. Suzumori, A. Sekizawa, E. Takeda et al., "Classification of factors involved in nonreportable results of noninvasive prenatal testing (NIPT) and prediction of success rate of second NIPT," Prenatal Diagnosis, vol. 39, no. 2, pp. 100-106, 2019.

[23] M. von Wolff, A. K. Schwartz, N. Bitterlich, P. Stute, and M. Fäh, "Only women's age and the duration of infertility are the prognostic factors for the success rate of natural cycle
IVF," Archives of Gynecology and Obstetrics, vol. 299, no. 3, pp. 883-889, 2019.

[24] N. Jahan, H. Talat, and W. T. Curry, "Agonist OX40 immunotherapy improves survival in glioma-bearing mice and is complementary with vaccination with irradiated GM-CSFexpressing tumor cells," Neuro-oncology, vol. 20, no. 1, pp. 44-54, 2018.

[25] E. Salimi, M. Karimi-Zarchi, S. A. Dastgheib et al., "Association of promoter region polymorphisms of IL-6 and IL-18 genes with risk of recurrent pregnancy loss: a systematic review and meta-analysis," Fetal and Pediatric Pathology, vol. 39, no. 4, pp. 346-359, 2020. 